\title{
Postural and Trunk Responses to Unexpected Perturbations Depend on the Velocity and Direction of Platform Motion
}

\author{
E. ZEMKOVÁ ${ }^{1,2}$, Z. KOVÁČIKOVÁ ${ }^{3}$, M. JELE $\check{N}^{1,2}$, K. NEUMANNOVÁ ${ }^{3}$, M. JANURA ${ }^{3}$ \\ ${ }^{1}$ Department of Sports Kinanthropology, Faculty of Physical Education and Sports, Comenius \\ University in Bratislava, Slovakia, ${ }^{2}$ Sports Technology Institute, Faculty of Electrical Engineering \\ and Information Technology, Slovak University of Technology in Bratislava, Slovakia, \\ ${ }^{3}$ Department of Natural Sciences in Kinanthropology, Faculty of Physical Culture, Palacký \\ University in Olomouc, Czech Republic
}

Received September 3, 2015

Accepted April 8, 2016

On-line July 15, 2016

\section{Summary}

This study compares postural and trunk responses to translating platform perturbations of varied velocities and directions. A group of 18 young and physically active subjects were exposed to a set of postural perturbations at varied velocities $(5,10,15$, and $20 \mathrm{~cm} / \mathrm{s}$ ) and directions of platform movement (forward, backward, left-lateral, and right-lateral). The center of pressure (CoP) displacement measurement, in addition to the trunk motion (representing the center of mass (COM) displacement), were both monitored. Results identified that the CoP displacement increased from slow to faster velocities of platform motion more widely in both anterior and posterior directions (50.4\% and $48.4 \%$ ) as compared to the CoM displacement (17.8\% and 14.9\%). However a greater increase in the peak CoM velocity $(70.3 \%$ and $69.6 \%$ ) and the peak CoM acceleration (60.5\% and $53.1 \%$ ) was observed. The values in the anterior and posterior direction only differed significantly at the highest velocity of platform motion (i.e. $20 \mathrm{~cm} / \mathrm{s}$ ). A similar tendency was observed in the medio-lateral direction, but there were no significant differences in any parameter in the left-lateral and right-lateral direction. The velocity of the platform motion highly correlated with peak velocity $(r=0.92-0.97, P<0.01)$ and moderately with amplitude of trunk displacement $(r=0.56-0.63, P<0.05)$. These findings indicate that the velocity of perturbation alters peak CoM velocity rather than the magnitude of CoM displacement. The effect of the direction of perturbations on the trunk response emerges only at a high velocity of platform motion, such that the peak CoM velocity and peak CoM acceleration are significantly greater in anterior than posterior direction.

\section{Key words}

Balance assessment - Center of mass - Center of pressure • Dynamic posturography $\bullet$ Translating platform

\section{Corresponding author}

E. Zemková, Faculty of Physical Education and Sports, Comenius University in Bratislava, Nábr. arm. gen. L. Svobodu 9, 81469 Bratislava, Slovakia. E-mail: Erika.zemkova@uniba.sk

\section{Introduction}

Experience indicates that in most cases, frequently used static posturography is not sensitive enough to differentiate athletic balance levels. This was corroborated by the study of Vuillerme et al. (2001) which highlighted that when visual cues were available, the CoP velocity and CoP range increased with the task difficulty (bipedal stance, unipedal stance on firm surface, unipedal stance on foam surface) when comparing gymnasts to athletes of other sports. Only when their vision was removed, a larger postural sway in the unipedal tasks was observed in the non-gymnasts when compared to the gymnasts.

Therefore, the combination of various testing conditions is usually utilized to increase the demands on the postural control system (Zemková 2011). These include varied surfaces (firm, foam), stances (bipedal, unipedal), feet position (semi-tandem, tandem), knee and hip angles (weight-bearing-leg: knee fully extended, knee 
flexed $10-20^{\circ}$; non-weight-bearing leg: knee flexed $90^{\circ}$, hip flexed $0^{\circ}$ or $45^{\circ}$ ), arm position (at the side, crossed over the chest, fixed on the hips), visual inputs (eyes open, fixed on stationary target, eyes closed), and so forth.

Lower sensitivity of static posturography is a consequence of multiple sensory inputs (visual, vestibular, and proprioceptive) involved in postural control. This system can compensate for a smaller impairment of balance in such a way that under normal conditions (quiet stance) no deficits in postural stability may be apparent. Under dynamic conditions (stance on an unstable surface) the control mechanisms are taxed to a substantially greater extent so that individual differences can be revealed (Zemková et al. 2005a). In sports, where the ability to maintain balance on an unstable support base is one of the limiting factors of performance, the dynamic posturography represents a more specific, and hence a more appropriate, alternative for the assessment of balance than systems allowing for monitoring of the CoP in static conditions (Zemková et al. 2005b).

Dynamic balance is the ability to maintain posture on an unstable support base. It is characterized by postural responses to external perturbations from a platform shifting in antero-posterior (A-P) and mediolateral (M-L) direction, or tilting toes up and down. Platform perturbations on some systems are unpredictable and are determined by the subject's positioning and sway movement. Other systems have a more predictable sinusoidal waveform, which remains constant regardless of subject positioning. Another example is the system that has a centrally pivoted platform with a tilt sensor. The stability of the platform is controlled by pressure in a pneumatic cushion under the platform and the tilt sensor monitors the deviation from the reference position. Some systems utilize a multiaxial platform similar to that of a BAPS board or wobble board that allows approximately $20^{\circ}$ of deflection in any direction. Novel systems are equipped with the trunk sensor applied to the subject's trunk which is capable of detecting trunk oscillations in A-P and M-L directions in erect as well as seated positions. These innovative features of posturography systems allow the assessment of various aspects of postural control.

The systems based on the force plate measure the vertical-ground reaction force and provide a means of computing the center of pressure $(\mathrm{CoP})$. The $\mathrm{CoP}$ is the weighted average of all the pressures over the surface area in contact with the ground. The net CoP is the integrated control variable of the center of mass (CoM) (Winter 1995). The displacement of the CoP can be utilized as a measure of stabilizing postural reactions in quiet standing, as well as in expected and unexpected perturbations.

The main disadvantage of the force platform analysis is that it measures the secondary consequences of swaying movements, not the movements themselves (Stevens and Tomlinson 1971). Increasing such CoP parameters as path length, area, displacement, or velocity does not necessarily link to postural instability. These variables may be indicative of underlying neural or sensorimotor dysfunction, but CoP movements may successfully stabilize the CoM. Subjects with high CoP velocity values may be quite stable in the sense that the CoP does not approach the limits of the base of support, but may require frequent postural corrections to achieve this degree of stability (Maki et al. 1990). And vice versa, the subject with lower CoP velocity can show greater path length and area. Thus, the interpretation of results often depends on the variable used.

To overcome these limitations, it has been suggested that the combined interpretation of CoP and CoM displacements provides a greater insight into the postural control than the CoP and the CoM taken separately (Murray et al. 1967, Spaepen et al. 1979, Winter 1995). In doing so, a biomechanical variable of CoP-CoM has been proposed. The major argument for using the CoP-CoM amplitude was that the difference between the $\mathrm{CoP}$ and $\mathrm{CoM}$ is proportional to the horizontal acceleration of the CoM during quiet standing (Winter 1995). The CoP-CoM variable has good reliability for both the test-retest and inter-rater studies, the ICC ranging from 0.89 to 0.93 in the A-P direction and from 0.74 to 0.79 in the M-L direction (Corriveau et al. 2001). However, an estimate of the CoM variable is somewhat difficult to obtain and has been crudely estimated, even if it seems to be related to postural imbalance (Winter 1995). Various techniques based on motion analysis or accelerometry recordings, while evaluating head, limb and trunk movements, have been used to provide additional data on postural control during quiet and perturbed standing. While research has established that postural response to the translating platform perturbation depends on its velocity (Runge et al. 1999, Bothner and Jensen 2001), amplitude (Horak et al. 1989) and displacement waveform (Brown et al. 2001), little is known regarding the underlying kinetics of 
trunk motion. Therefore, we devised an experiment to investigate the relationship between the velocity and the direction of platform motion and the CoP and CoM displacement, while the other characteristics underlying the translating platform perturbation (e.g. the displacement waveform or amplitude) remained constant. We hypothesized that the magnitude of the CoP displacement increases proportionally with escalating the velocity of the platform motion, whereas the CoM displacement also increases, but to a lesser extent, in addition to the differential effects of the direction of platform motion on the CoM variables (i.e. peak velocity and acceleration). Verification of this hypothesis was accomplished by comparing postural ( $\mathrm{CoP})$ and trunk $(\mathrm{CoM})$ responses to unexpected perturbations induced by

a

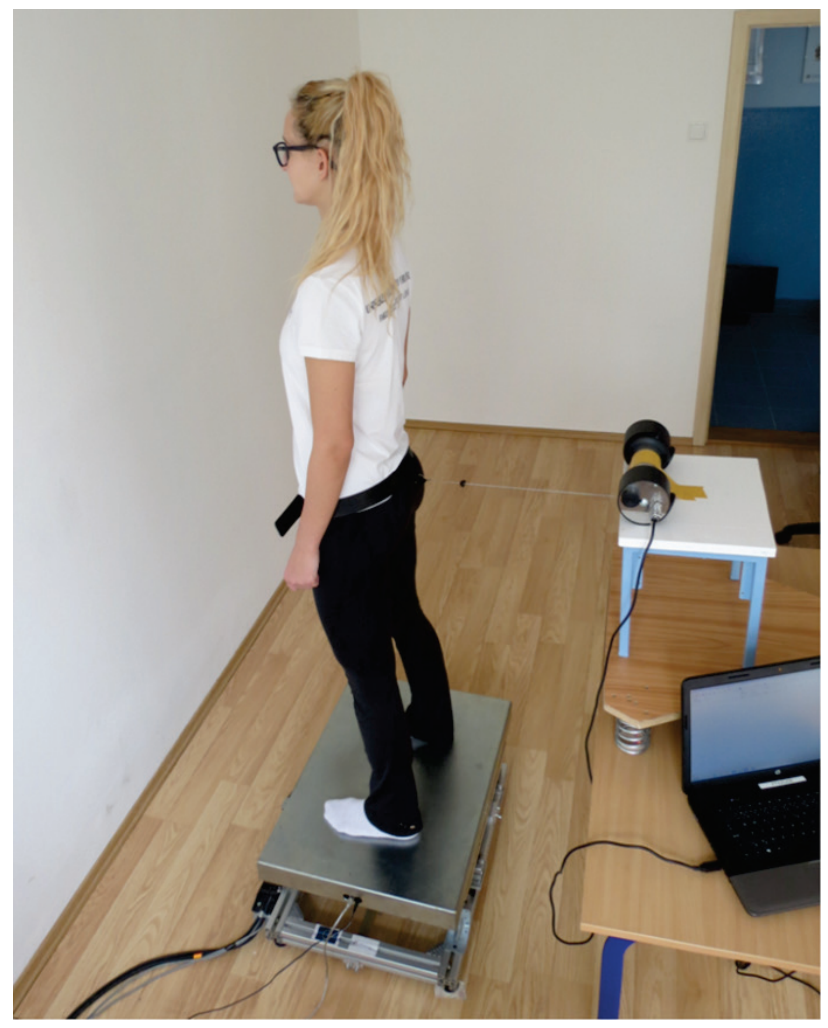

varied velocities and directions of platform translation.

\section{Methods}

\section{Subjects}

A group of 18 young and physically active subjects (11 males of age $22.7 \pm 2.4$ years, height $178.5 \pm 9.9 \mathrm{~cm}$, weight $76.9 \pm 7.2 \mathrm{~kg}$, and 7 females of age $23.0 \pm 3.1$ years, height $171.3 \pm 8.9 \mathrm{~cm}$, weight $63.6 \pm 10.2 \mathrm{~kg}$ ) volunteered to participate in the study. All participants were informed of the procedures and the main purpose of the study. The procedures presented were in accordance with the ethical standards on human experimentation stated in compliance with the Helsinki Declaration.
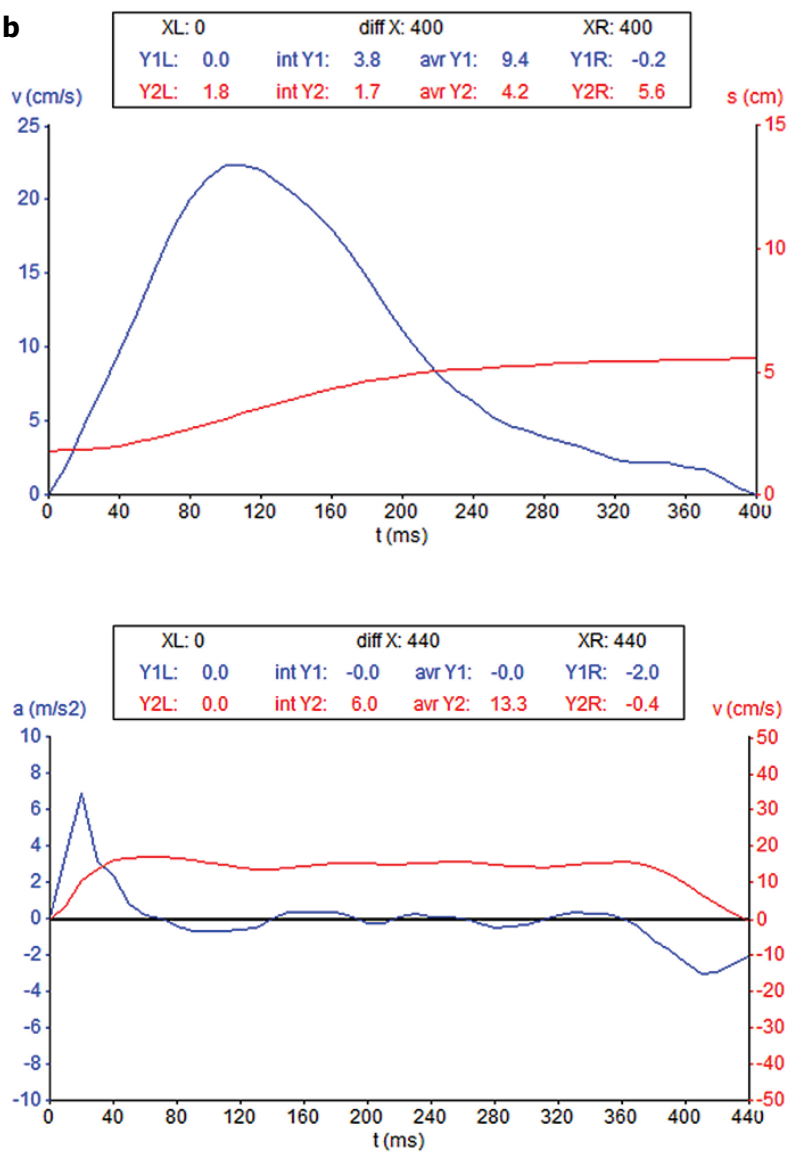

Fig. 1. (a) FiTRO Dyne Premium and FiTRO Dynamic Posturography system and an example of on-line display (b - above) of peak velocity and range of trunk movement and ( $\mathbf{b}-\mathbf{b e l o w})$ velocity of platform translation with corresponding acceleration.

Participants were instructed to stand barefoot on a force platform, with their arms relaxed comfortably at their sides. They were required to stand in an upright posture with their feet abducted $10^{\circ}$ and their heels separated medio-laterally by a distance of $6 \mathrm{~cm}$. They were not previously informed regarding the direction and timing of perturbations. A signal from the computer triggered a random motion of the platform, thus the subject received no cues as to when the perturbation would occur. The platform motion produced a sudden change in the external forces acting on the subject, leading to a displacement of the subject's center of 
pressure. The perturbation caused only a postural sway response, i.e. the subject did not need to take a step to maintain balance. The perturbations were produced randomly in four directions, i.e. forward, backward, leftlateral, and right-lateral, respectively. Each test consisted of two trials with the most enhanced results recorded for evaluation.

Parameters of balance, the peak CoP displacement and time to peak $\mathrm{CoP}$ displacement, were recorded 5-s before, during, and 5-s following the sudden motion of the platform using the FiTRO Dynamic Posturography system (FiTRONiC, Slovakia) (Fig. 1a, $\mathrm{b}$-below). Ground reaction forces were recorded at a sampling frequency of $100 \mathrm{~Hz}$. The force plate was placed over a moving device that produces side-to-side movements at a predefined velocity and amplitude. Our previous experiments identified that the platform is able to achieve maximal velocity of $20 \mathrm{~cm} / \mathrm{s}$ at least at $6 \mathrm{~cm}$ distance (Zemková et al. 2015). Therefore, a constant range of $6 \mathrm{~cm}$ was determined for all velocities utilized $(5 \mathrm{~cm} / \mathrm{s}, \quad 10 \mathrm{~cm} / \mathrm{s}, \quad 15 \mathrm{~cm} / \mathrm{s}, \quad$ and $20 \mathrm{~cm} / \mathrm{s})$. Peak acceleration was approximately $6 \mathrm{~m} / \mathrm{s}^{2}$.

Concurrently with measurement of dynamic balance, trunk movement representing roughly the center of mass (CoM) movement was also monitored using the FiTRO Dyne Premium (FiTRONiC, Slovakia) (Fig. 1a, $\mathrm{b}-$ above). This system consists of a precise analogue rotary sensor coupled to a reel. When pulling on the tether, the reel is wound while a sensor measures the velocity. Rewinding of the reel is guaranteed by a string producing force of about $2 \mathrm{~N}$. The signal is passed through a 12-bit analogue-to-digital convertor and sent to a PC by a USB cable. The included comprehensive software was utilized to collect, calculate, and display real-time basic parameters involved in trunk movement. Peak values of velocity, acceleration and amplitude of trunk movement in each direction were selected for analysis. The device was placed on the table and attached to the belt by a nylon tether.

\section{Statistical analysis}

Data analyses were performed using statistical program SPSS for Windows, version 18.0 (SPSS, Inc., Chicago, IL, USA). Ordinary statistical methods including mean and standard deviations were utilized. Data was analysed using a repeated measure analyses of variance (ANOVA) for factors of velocity $(5 \mathrm{~cm} / \mathrm{s}$, $10 \mathrm{~cm} / \mathrm{s}, 15 \mathrm{~cm} / \mathrm{s}$, and $20 \mathrm{~cm} / \mathrm{s}$ ) and direction of platform motion (forward, backward, left-lateral, and right-lateral).
Post-hoc tests were utilized to determine significant main and interaction effects. The Student's paired t-tests were employed to determine the statistical significance of difference in postural and trunk responses to varied perturbations. Depending on a number of comparisons, levels of significance set at 0.05 were adjusted using Bonferroni correction. The relationships between values of postural and trunk stability variables were determined by Pearson's correlation coefficients. Tests were considered significant at a level of $P<0.05$.

\section{Results}

The repeated measurement analysis identified a significant main effect for the velocity of platform motion on the CoP displacement $\left(F_{4,68}=21.73, P<0.01\right)$, but not on the CoM displacement $\left(F_{4,68}=0.85, P=0.52\right)$, such that $\mathrm{CoP}$ displacement was greater for faster perturbations. The ANOVA also revealed the main effects on the CoM velocity $\left(F_{4,68}=54.92, P<0.001\right)$ and the CoM acceleration $\left(F_{4,68}=40.27, P<0.001\right)$.

In addition, the ANOVA identified significant effects for the direction of platform motion on the CoM velocity $\left(F_{4,68}=11.51, P<0.05\right)$ and the CoM acceleration $\left(F_{4,68}=9.46, P<0.05\right)$, but not on the CoM displacement $\left(F_{4,68}=0.41, \quad P=0.83\right)$ and the CoP displacement $\left(F_{4,68}=0.58, P=0.66\right)$.

However, no significant direction $\mathrm{x}$ velocity interaction was identified $\left(F_{2,16}=1.07, P=0.35\right)$, although the direction effect tended to be greater for faster perturbations $\left(F_{2,16}=2.92, P=0.068\right)$.

As can be seen in Figures 2a and b, the CoP displacement increased from slow to fast velocities of platform motion (from $5 \mathrm{~cm} / \mathrm{s}$ to $20 \mathrm{~cm} / \mathrm{s}$ ) more widely in both anterior and posterior directions $(50.4 \%$ and $48.4 \%$, respectively) when compared to CoM displacement $(17.8 \%$ and $14.9 \%$, respectively). However, neither CoP nor CoM displacement differed significantly in either the anterior or posterior direction.

In comparison with the amplitude of CoM displacement, there was a steeper increase in peak CoM velocity with increasing the velocity of platform motion in both anterior and posterior directions $(70.3 \%$ and $69.6 \%$, respectively). A similar trend was observed for peak CoM acceleration $(60.5 \%$ and $53.1 \%$, respectively). Although these values were higher in the anterior than the posterior direction, significant differences were only identified at the maximum velocity of platform motion utilized in the present study (i.e. 
$20 \mathrm{~cm} / \mathrm{s}$ ) (Fig. 3a, b).

As expected, there were no significant differences in CoP displacement, CoM displacement, $\mathrm{CoM}$ velocity and $\mathrm{CoM}$ acceleration in left-lateral and right-lateral direction at all velocities of platform motion $(P>0.05)$. An increase in $\mathrm{CoP}$ displacement with increasing the platform motion was greater when compared to CoM displacement $(46.5 \%$ and $12.7 \%$, respectively). Nevertheless, a steeper increase in peak $\mathrm{CoP}$ velocity and peak $\mathrm{CoP}$ acceleration was observed (66.1\% and $49.8 \%$, respectively).

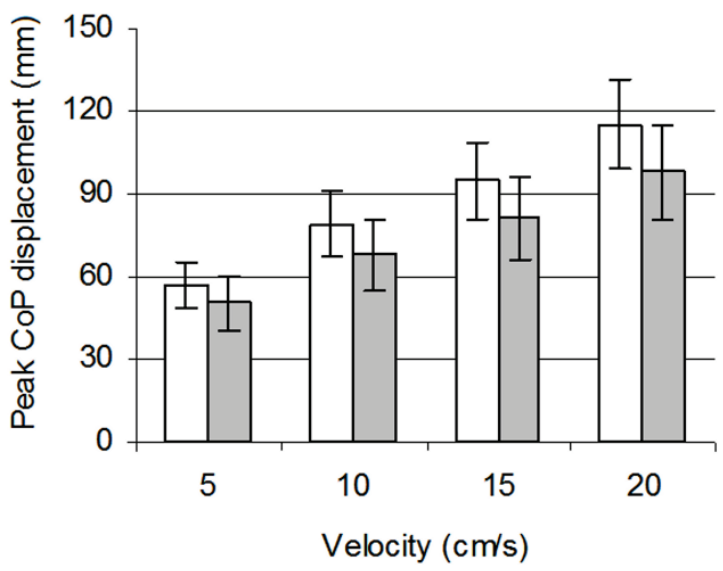

$\square$ Anterior $\square$ Posterior

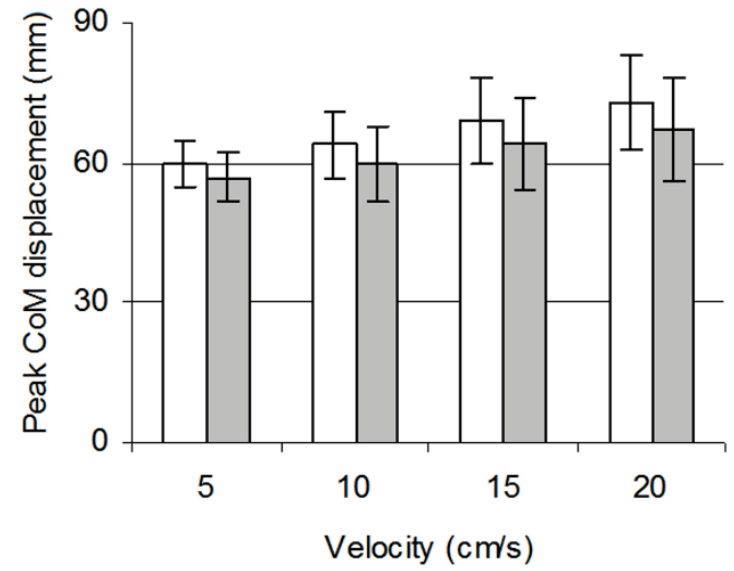

$\square$ Anterior $\square$ Posterior

Fig. 2. a) The CoP displacement and b) the CoM displacement in anterior and posterior directions after translating platform perturbations of different velocities.

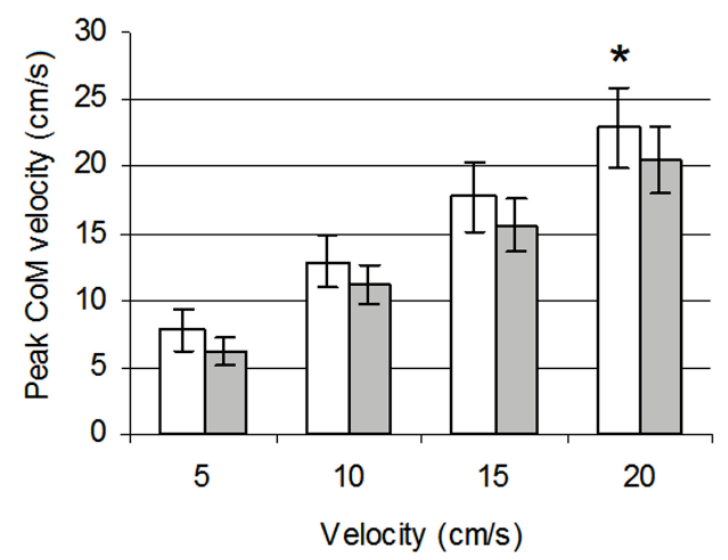

$\square$ Anterior $\square$ Posterior b

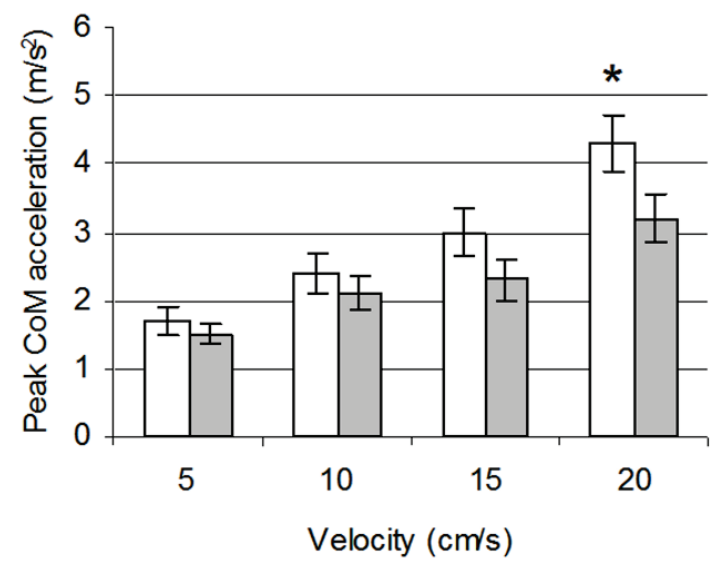

$\square$ Anterior $\square$ Posterior

Fig. 3. a) Peak CoM velocity and b) peak CoM acceleration in anterior and posterior directions after translating platform perturbations of different velocities.

Furthermore, the velocity of the platform motion highly correlated with the peak velocity of trunk motion at all velocities applied $(5-20 \mathrm{~cm} / \mathrm{s})$ in anterior (r=0.95-0.97, $P<0.01$ ), posterior $(\mathrm{r}=0.94-0.97, P<0.01)$, and both lateral directions $(\mathrm{r}=0.92-0.96, P<0.01)$. There were also moderate correlations between the velocity of platform motion and the amplitude of trunk displacement in anterior $(\mathrm{r}=0.59-0.63, P<0.05)$, posterior $(\mathrm{r}=0.57-0.61$, $P<0.05)$, and both lateral directions $(\mathrm{r}=0.56-0.61$, $P<0.05)$.

\section{Discussion}

The objective of this study was to investigate 
whether balance disturbances of the same magnitude and waveform of displacement, but with differing velocities and directions of platform motion, created varied postural and trunk responses. Our study extends that of Runge et al. (1999) and Bothner and Jensen (2001) who have revealed that the underlying kinetics of postural recovery are dependent upon the velocity of platform translation. We have demonstrated that a balance disturbance, equivalent in magnitude and waveform of displacement, can also lead to differences in trunk response if the velocity of platform motion is varied.

Perturbation velocity altered the peak CoM velocity and peak CoM acceleration, but did not significantly affect the amplitude of CoM movement. The increase in peak CoM velocity was proportional to the increasing velocity of platform motion, whereas the magnitude of CoM displacement did not alter significantly and followed a similar pattern to the amplitude of platform motion. The proportional increase in peak CoM velocity with increasing velocity of platform motion implies a platform-induced influence to the horizontal velocity of the CoM. In other words, it appears that the underlying trunk response to perturbations is dependent upon the velocity of platform translation. Indeed, a post-hoc test of correlation between the velocity of trunk motion and the velocity of platform motion confirmed our hypothesis $(\mathrm{r}=0.92-0.97, P<0.01)$. The magnitude of the trunk response moderately correlated with the velocity of platform motion $(\mathrm{r}=0.56-0.63, P<0.05)$ meaning that the velocity of the perturbation also altered the amplitude of CoM movement, but to a lesser extent.

It appears that increasing the magnitude of the CoP displacement does not necessarily link to trunk instability. This variable may be an indication of underlying balance disorders, but $\mathrm{CoP}$ movements may successfully stabilize the CoM. Subjects with high CoM velocity values may be quite stable in terms of small range of CoM displacement. We have observed that the magnitude of the trunk response (about $6-7 \mathrm{~cm}$ ) was similar to the amplitude of the platform displacement $(6 \mathrm{~cm})$. On the other hand, Horak et al. (1989) demonstrated that the magnitude of the postural response is scaled to the amplitude of platform displacement.

In the case of trunk response to perturbations, we also identified that the velocity of perturbation alters the velocity of trunk motion rather than the magnitude of its displacement. This paradigm is representative of the type of disturbances experienced in real life. Unexpected sudden shifts of a support surface, rather than the magnitude of its translation, may cause trunk stability impairment. This is contrary to CoP displacement, which escalates proportionally with the increasing velocity of platform motion.

Our findings also suggest that the effects of perturbation direction only emerge in high severity perturbation conditions (i.e. $20 \mathrm{~cm} / \mathrm{s}$ of platform motion) which indicate that within these conditions, peak CoM velocity and peak CoM acceleration is greater in the anterior rather than posterior direction.

In the $\mathrm{A} / \mathrm{P}$ direction, both an ankle and a hip strategy have been described (Horak and Nashner 1986). The ankle strategy applies in quiet stance and during small perturbations and predicts that the ankle plantarflexors/dorsiflexors alone act to control the inverted pendulum. In more perturbed situations, or when the ankle muscles cannot act, a hip strategy would respond to flex the hip, thus moving CoM posteriorly, or to extend the hip to move the CoM anteriorly.

The classical work by Nashner (1977) could explain postural and possibly also trunk responses to perturbations applied by a translating platform. The author demonstrated that when the platform moved backwards, the gastrocnemii and hamstrings had the most common response with latencies of 100-120 ms after the onset of platform translation. This response by the posterior muscles was matched by a common response by the anterior muscles (tibialis anterior and rectus femoris) when the platform was translated forward. However, there were a small percentage of exceptions. In $18 \%$ of the imposed dorsiflexor trials, only the gastrocnemii responded, and in $22 \%$ of the plantarflexor perturbations, all four muscles responded. In a few trials only the gastrocnemii and tibialis anterior responded to a backward translation and the gastrocnemii also responded with the normal tibialis anterior/rectus femoris to a forward translation.

Nashner (1982) suggested that there was a bottom-up sequence in the initial responses. For a backward translation of the platform, the gastrocnemii responded first, followed by the hamstrings, then the erector spinae. For a forward platform movement, the sequence was tibialis anterior-rectus femoris-abdominals. Thus it appears the CNS recognizes the need to stabilize the joint closest to perturbation first, followed by the knee, hip, and spine. Because the responses radiated from the ankle towards the body's CoM, those responses were described as an 'ankle strategy'. An alternate strategy, 
called a 'hip strategy', was identified when the ankle muscles were unable to respond. When the platform was displaced in a backward direction, the CNS responded with a strong hip flexor (abdominal + rectus femoris) pattern. A hip flexor moment is more effective in shifting the body CoM than an ankle strategy. The reverse response by the extensors (hamstrings + erector spinae) was evident when the platform was translated forward.

The effect of the direction of unexpected horizontal perturbations of stance on the organization of automatic postural responses was studied in human subjects (Moore et al. 1988). These authors suggest that composition of postural responses is a complex process that includes perturbation direction as a continuous variable. Using the FiTRO Dynamic Posturography system, Kováčiková et al. (2014) reported more profound balance impairment at fast platform motion $(20 \mathrm{~cm} / \mathrm{s})$ in both lateral directions, as compared to the anteriorposterior direction. In lateral perturbations, significantly greater re-stabilization time on the preferred limb side than on the non-preferred limb side was also identified (Kováčiková et al. 2015). The authors suggested that the observed shorter re-stabilization time at the non-preferred limb side, was due to the stabilization role of the nonpreferred limb. Our present work contributed to these results by identifying significant differences in trunk response to translating platform perturbations in anterior and posterior direction, however only at the highest velocity applied of $20 \mathrm{~cm} / \mathrm{s}$. Thus, both the velocity and direction of platform motion play a role in the trunk response to perturbations.

Overall, our findings revealed that velocity of platform motion differentially influenced the postural and trunk responses to the perturbations. The CoP displacement from slow to fast velocities of platform motion increased more widely than CoM displacement. However, peak CoM velocity increased proportionally with increasing the velocity of platform motion and these values were slightly higher than those of the platform motion. A similar tendency for peak CoM acceleration was observed, although these values were lower than the acceleration of platform motion. The effect of direction of platform motion on the trunk response to the perturbation emerged only for the high velocity of platform motion (i.e. $20 \mathrm{~cm} / \mathrm{s}$ ). The peak CoM velocity and peak CoM acceleration were significantly higher in anterior than posterior direction. As expected, there were no significant differences in CoP displacement, CoM displacement, $\mathrm{CoM}$ velocity and $\mathrm{CoM}$ acceleration in left-lateral and right-lateral direction at all velocities of platform motion. The values altered with increasing velocity of platform motion similarly to those in antero-posterior direction. Most importantly, velocity of platform motion highly correlated with peak velocity of the CoM motion in anterior, posterior and both lateral directions. There were also moderate correlations between velocity of platform motion and amplitude of CoM displacement in anterior, posterior, and both lateral directions.

It may be concluded that velocity of perturbation alters peak CoM velocity rather than the magnitude of CoM displacement, and that the effect of direction of perturbation on the trunk response emerges only for the high velocity of platform motion. This raises the question as to whether increases in the amplitude of trunk movement under translating platform motion is necessarily an indication of impaired trunk and/or postural stability. Therefore, future work is warranted to investigate the trunk response to perturbations of varied velocities and directions in older adults and those with impaired balance functions.

\section{Conflict of Interest}

There is no conflict of interest.

\section{Acknowledgements}

This work was supported by the Slovak Research and Development Agency under the contract Nos. SK-CZ2013-0131 and 7AMB14SK073 as well as by the Scientific Grant Agency of the Ministry of Education of Slovak Republic and the Slovak Academy of Sciences under the contract No. 1/0373/14.

\section{References}

BOTHNER KE, JENSEN JL: How do non-muscular torques contribute to the kinetics of postural recovery following a support surface translation? J Biomechanics 34: 245-250, 2001.

BROWN LA, JENSEN JL, KORFF T, WOOLLACOTT MH: The translating platform paradigm: perturbation displacement waveform alters the postural response. Gait Posture 14: 256-263, 2001. 
CORRIVEAU H, HÉBERT R, PRINCE F, RAICHE M: Postural control in the elderly: an analysis of test-retest and inter-rater reliability of the COP-COM variable. Arch Phys Med Rehabil 82: 80-85, 2001.

HORAK FB, DIENER HC, NASHNER LM: Influence of central set on human postural responses. J Neurophys 62: 841-852, 1989.

HORAK FB, NASHNER LM: Central programming of postural movements: adaptation to altered support surface configurations. J Neurophysiol 55: 1369-1381, 1986.

KOVÁČIKOVÁ Z, ZEMKOVÁ E, JELEŇ M, VILMAN T: More profound balance impairment at fast platform shift occurs in lateral than anterior-posterior direction. 1st Clinical Movement Analysis World Conference. Rome: $232,2014$.

KOVÁČIKOVÁ Z, ZEMKOVÁ E, NEUMANNOVÁ K, JELEŇ M, JELEN K, JANURA M: The role of lateral preference of lower limbs in a postural stabilization task. Neuro Endocrinol Lett 36: 91-95, 2015.

MAKI BE, HOLLIDAY PJ, FERNIE GR: Aging and postural control. A comparison of spontaneous- and inducedsway balance tests. J Am Geriatr Soc 38: 1-9, 1990.

MOORE SP, RUSHMER DS, WINDUS SL, NASHNER LM: Human automatic postural responses: responses to horizontal perturbations of stance in multiple directions. Exp Brain Res 73: 648-658, 1988.

MURRAY MP, SEIREG A, SCHOLZ RC: Center of gravity, center of pressure, and supportive forces during human activities. J Appl Physiol 23: 831-838, 1967.

NASHNER LM: Adaptations of human movement to altered environments. Trends Neurosci 5: 358-361, 1982.

NASHNER LM: Fixed patterns of rapid postural responses among leg muscles during stance. Exp Brain Res 30: 13-24, 1977.

RUNGE CF, SHUPERT CL, HORAK FB, ZAJAC FE: Ankle and hip postural strategies defined by joint torques. Gait Posture 10: 161-170, 1999.

SPAEPEN AJ, PEERAER L, WILLEMS EJ: Center of gravity and the center of pressure in stabilometric studies. Agressologie 20: 117-118, 1979.

STEVENS DL, TOMLINSON GE: Measurement of human postural sway. Proc R Soc Med 64: 653-654, 1971.

VUILLERME N, DANION F, MARIN L, BOYADJIAN A, PRIEUR JM, WEISE I, NOUGIER V: The effect of expertise in gymnastics on postural control. Neurosci Lett 303: 83-86, 2001.

WINTER DA: A.B.C. of Balance During Standing and Walking. Waterloo Biomechanics, Waterloo (Ont), 1995.

ZEMKOVÁ E: Assessment of balance: Science and reality (Invited review). Serb J Sports Sci 5: 127-139, 2011.

ZEMKOVÁ E, HAMAR D, BÖHMEROVÁ L': The dynamic balance - reliability and methodological issues of novel computerized posturography system. Med Sport 9: 76-82, 2005a.

ZEMKOVÁ E, VIITASALO J, HANNOLA H, BLOMQVIST M, KONTTINEN N, MONONEN K, PAHTAJA V, SIRVIÖ R: Sensory Organisation Test in diagnostics of post-exercise postural stability of athletes. Sport Sci 1: 26-32, 2005b.

ZEMKOVÁ E, KOVÁČIKOVÁ Z, JELEŇ M, SVOBODA Z, JANURA M: Methodological issues of dynamic posturography specific to the velocity and the displacement of the platform perturbation. Proceedings of Scientific Studies "From Research to Practice”. Bratislava, 1-10, 2015. 